\title{
JLECOV
}

\section{Bitcoin kaotik analizi ve fiyat tahmin modeli önerisi}

\section{Bitcoin chaotic analysis: A price forecasting model proposal}

\section{H. Nazan Çağlar}

Dr. Öğr. Üyesi, İstanbul Kültür Üniversitesi, İktisadi ve İdari Bilimler Fakültesi, Girişimcilik Bölümü, Türkiye, e-mail: ncaglar@iku.edu.tr

\section{Özet}

Kaos Teorisi, doğrusal olmayan dinamik sistemlerin davranışlarını tanımlar ve ekonomi alanında pek çok verinin modellenmesinde kullanılır. Kaos teori, sistemin doğrusal olmayan ve deterministik bir süreç olduğu varsayımlarına dayanır. Doğrusal modeller, ekonometrik sistemleri karmaşıklıklarını ortaya çıkarmakta yetersiz kalmaktadır. Bu çalışmanın amacı, Bitcoin günlük fiyatlarının zamana bağlı doğrusal olmayan dinamik bir sistem tarafından üretilip üretilmediğini araştırmak ve sistemin uzun vadede geleceğe yönelik tahmin yeteneğini araştırmak ve bir tahminleme modeli oluşturmaktır. Birçok ekonomik veri serisinin kaotik davranış gösterdiği bilinmektedir. Bu çalışmada, Bitcoin fiyatlarının kaotik yapısı incelenmiş ve regresyon yöntemi kullanılarak tahmin modeli kurulmuştur. Diğer bir ifadeyle amaç, Bitcoin fiyatlarının getirilerinin kaotik bir davranış gösterip göstermediğini ortaya koyarak elde edilen gömme (embedding) boyutuna bağlı olarak regresyon yöntemini kullanarak tahmin modeli oluşturmaktır. Çalışmada, 2021 Şubat - 2021 Kasım döneminde günlük kapanış fiyatı ( \$) veri olarak kullanılmıştır. (URL-1,2021)

Anahtar kelimeler: Kaos Teorisi, Faz Uzayı, Zaman Serisi, Gömme (Embedding ) Boyutu, Doğrusal Olmayan Kaotik Sistem, Bitcoin, Regresyon Modeli

JEL kodları: C10, C22, C51, C53, G10

\begin{abstract}
Chaos theory describes the behavior of nonlinear dynamic systems and has been used in solving a wide range of problems in economics. The theory of Chaos is based on the assumption that the underlying system is a nonlinear deterministic process. On the other hand, linear models, which are proven to be insufficient in revealing the complexities of economic systems, are not seen as an appropriate modelling approach for analyzing economic data which show chaotic behavior. The focus of this study is two-fold; the first objective is to investigate whether

Citation/Atıf: Çağlar, H. N., (2022). Bitcoin kaotik analizi ve fiyat tahmin modeli önerisi. Journal of Life Economics. 9(1):33-39, DOI: 10.15637/jlecon.9.1.03


Bitcoin's non-linear daily price change shows a chaotic behavior. The second objective is to investigate whether the system is suitable for a long-term forecast. The current research investigates both objectives by conducting a prediction model by using a regression model which depends on the embedding size. In order to capture the rich dynamic information hidden in the price changes of Bitcoin, we use the daily closing prices (\$) of Bitcoin between the two periods February 2021 and November 2021. The data for such analysis was obtained from marketwach.com.

Keywords: Chaos Theory, Phase Space, Time Series, Embedding Dimension, Nonlinear Chaotic System, Bitcoin, Regression Model.

JEL cods: C10, C22, C51, C53, G10

\section{GİRiş}

Kaos teorisi kimya, fizik, mühendislik, ekoloji ve ekonomide çok çeşitli problemlerin çözümünde kullanılmaktadır. Geçen yüzyıl boyunca ekonomistler, iş çevrimi de dahil olmak üzere ekonomik çevrimler biçiminde farklı dinamik davranışların varlığını öne sürdüler. Kaos nedir sorusu, genel olarak üç koşulla karakterize edilen düzensiz salınımlı bir süreç olarak cevaplanmıştır. Bu koşullar; düzensiz periyodiklik, başlangıç koşullarına ve parametreye duyarlılık, öngörülebilirlik eksikliği olarak belirtilir.

Kaos, temel alınan sistemin doğrusal olmayan ve deterministik bir süreç olduğu varsayımlarına dayanır. Bununla birlikte, doğrusal modellerin ekonomik sistemin karmaşıklığını yakalamada başarısız olduğunu kanıtlayan bazı sonuçlar vardır. Doğrusal olmayan analiz başlangıçta fizik ve doğa bilimlerinde geliştirilmiş olsa da, doğrusal modeller bu alanlardaki her sorunu açılayamadığı için finans ve ekonomiye de uygulanmıştır.

Kaos teorisi, doğrusal olmayan dinamikleri incelemede yeni bir yöntem sunduğundan, doğrusal olmayan dinamiklerin ekonomideki uygulamalarının çoğu, makroekonomide büyüme ve iş çevrimlerini modellemeye odaklanmıştır.( Gangopadhyay, P. et al., 2011) Finansal piyasaların kaotik ve karmaşık yapısını anlamak için geçmişin katı bilimsel paradigmaları ile çalışmak yerine yeni matematiksel teknikler kullanılmalıdır. (Nichols, Nancy A., 1993)
Peters ( Peters,E.,1991) kitabında, kaos teorisinin, piyasayı birbirine bağlı karmaşık bir sistem gibi ele alan yeni bir tür piyasa paradigması yaratmanın anahtarının gerçekten elinde olabileceğini savunuyor. Kitabın girişinde şöyle yazıyor: "Etkin piyasa hipotezi, yatırımciların rasyonel, sıralı ve düzenli olduğunu varsayar. Matematiği tek bir çözümle basit doğrusal diferansiyel denklemlere indirgeyen bir yatırım davranışı modelidir. Ancak, piyasalar düzenli veya basit değildir. Dağınık ve karmaşıktırlar."

Çalışmaların çoğu, önceki dönem getirilerinin sonraki dönem akışları üzerindeki önemini ve önceki dönemlere ait riske göre düzeltilmiş getirilerin sıralanmasının cari dönem akışları üzerindeki önemini ortaya koymuştur. (Clark,A.,2006:50)Zaman Serilerinin kaotik davranışlarının analizi üzerine farklı alanlarda pek çok çalışma vardır. (Albostan ,A. , Önsöz, B. 2015 ) Ayrıca Bitcoin modelleri üzerine de çalışmalar mevcuttur. ( Sakız,B., Gencer,A.H. (2017). (İşeri, M.,Caglar H.,Caglar,N. 2008)

Dijital para birimi Bitcoin ilk olarak 2008 yılında Satoshi Nakamoto'nin (URL-2,2021) elektronik para birimi ve eşten eşe (peer-to-peer) transfer konusundaki makalesi ile ortaya atılan bir sistemdir. Satoshi'nin fikri, 2009 yllında Bitcoin yatırım aracı olarak ortaya çıkmıştır. Bitcoin'in değerini piyasadaki arz ve talep koşulları belirler. Bu çalışmada, Bitcoin fiyatlarına ait serinin kaotik analizi yapılmış ve bulunan sonuca göre regresyon modeli kullanılarak ileriye yönelik 
olarak tahminlenebilirliği araştırılmıştır.

\section{KAOTIK ANALIZ}

\subsection{Faz Uzayı ve Gömme ( Embedding) Boyutu}

Kaotik davranış gösteren zaman serileri ve kaos teorisi yöntemlerinin arkasındaki önemli temellerden biri, gömme teoremidir. (Takens F., 1981) Tek bir ölçülen değişken $x(n)=x\left(t_{0}+n \tau\right)$ ile $t_{0}$ başlangıç zamanı ve $\tau$ örnekleme zamanı ve bunun gecikmelerinin kullanımınin N-boyutlu uzayı sağladığını gösterir. X(t) serisi kendi içerisinde birçok dinamik davranışı içerebilir. Gözlemlenen sistemdeki dinamik davranışlar için $\mathrm{N}$ boyutlu durum vektörleri $X(t)$ aşağıdaki şekilde tanimlanır.

$X(t)=[x(t), x(t+\tau), \ldots, x(t+(N-1) \tau)]$

burada $x(t)$, $t$ zamanındaki zaman serisinin bir değeridir, $\tau$ uygun bir zaman gecikmesidir (örnekleme zamanı) ve $\mathrm{N}$ gömme boyutudur. $\mathrm{Bu}$ vektör, $N$ yeterince büyük olduğunda, doğrusal olmayan dinamikleri tam olarak temsil eder. Gömme teoremi, bir sistem üzerindeki davran1Şın tam bilgisinin herhangi bir ölçümün zaman serisinde yer aldığını ve zaman serisinden çok değişkenli faz uzayı için yeniden yapılandırma oluşturulabileceğini gösterir. Durum uzayını zaman gecikmesinde $\tau$ gerçekleştirmek için gömme boyutu $\mathrm{N}$ gereklidir.

Tek boyutlu zaman serileri $X\left(t_{0}\right), X\left(t_{1}\right), \ldots, X\left(t_{i}\right), \ldots$ , $X\left(t_{n}\right)$ varsayalım ve $m$ boyutlu faz uzayının bir faz tipine genişletilir,

$$
\begin{array}{lccccc}
X\left(t_{0}\right) & X\left(t_{1}\right) & \ldots & X\left(t_{i}\right) & \ldots & X\left(t_{n}+(m-1) \tau\right) \\
X\left(t_{0}+\tau\right) & X\left(t_{1}+\tau\right) & \ldots & X\left(t_{i}+\tau\right) & \ldots & X\left(t_{n}+(m-2) \tau\right) \\
X\left(t_{0}+2 \tau\right) & X\left(t_{1}+2 \tau\right) & \ldots & X\left(t_{i}+2 \tau\right) & \ldots & X\left(t_{n}+(m-3) \tau\right)
\end{array}
$$

$$
\begin{array}{cccc}
\mathrm{X}\left(\mathrm{t}_{0}+(\mathrm{m}-1) \tau\right) & \mathrm{X}\left(\mathrm{t}_{1}+(\mathrm{m}-1) \tau\right) & \ldots & \mathrm{X}\left(\mathrm{t}_{\mathrm{i}}+(\mathrm{m}-1) \tau\right) \\
\ldots & \mathrm{X}\left(\mathrm{t}_{\mathrm{n}}\right) & &
\end{array}
$$

Burada $\tau=\mathrm{k} \Delta \mathrm{t}(\mathrm{k}=1,2, \ldots)$ gecikme zamanıdır ve denklemdeki her satırdan faz uzayının bir faz noktası oluşturulur (2.2). Her faz noktası $X\left(t_{i}\right) m$ ağırlığa sahiptir, $X\left(t_{i}\right), X\left(t_{i}+\tau\right), \ldots, X\left(t_{i}+(m-1) \tau\right)$.
m-Boyutlu faz uzayının her bir faz noktası, kesinlikle anlık bir durumu içerir ve noktanın faz uzayının yörüngesi, faz noktasının bağlantı çizgisinden oluşurken, zamanla sistem durumu evrimini sergiler. Daha sonra sistem dinamiği daha fazla boyutta faz uzayında incelenebilir. Sistemin gömme boyutunun belirlenmesi amacıyla korelasyon boyutu yöntemi kullanılır.

\subsection{Korelasyon Boyutu ( Correlation Dimensi- on)}

Korelasyon boyutu en temel bilinen kaos testidir. Yöntem, gözlemlenen zaman serisi verilerinden boyutu belirlemeye çalışır. Bir diğer ifadeyle, sistemin gömme boyutunun belirlenmesinde kullanılan en temel yöntem korelasyon boyutudur. Grassberger ve Procaccia tarafından önerilen algoritma (Grassberger P ve Procaccia I., 1983), çekicinin boyutunu tahmin etmek için güç yasası davranışı kullanılan bir korelasyon integralinin hesaplanmasını içerir. $\mathrm{m}$ boyutlu faz uzayında bir çift faz noktası

$$
\begin{aligned}
& X_{m}\left(t_{i}\right)=\left(X\left(t_{i}\right), X\left(t_{i}+\tau\right), X\left(t_{i}+2 \tau\right), \ldots X\left(t_{i}+(m-1) \tau\right)\right. \\
& X_{m}\left(t_{j}\right)=\left(X\left(t_{j}\right), X\left(t_{j}+\tau\right), X\left(t_{j}+2 \tau\right), \ldots X\left(t_{j}+(m-1) \tau\right)\right.
\end{aligned}
$$

Uzaklıkları $r_{i j}=X_{m}\left(t_{i}\right)-X_{m}\left(t_{j}\right)$ olduğunda. Kritik bir mesafe $r$ verildiğinde, korelasyon integrali şu şekilde tanımlanır:

$$
C(r, m)=\frac{1}{N(N-1)} \sum_{i, j=1(i \neq j)}^{N} \theta\left(r-\left\|X_{i}-X_{j}\right\|\right), 1 \leq \mathrm{i} \leq \mathrm{j} \leq \mathrm{N}
$$

$X_{i}, X_{j}$ vektörleri arasındaki uzaklık $\left\|X_{i}, X_{j}\right\|$ öklidien uzaklıktır. Burada $\theta$, Heaviside fonksiyonudur. $\mathrm{z}=\mathrm{r}-\left\|X_{i}, X_{j}\right\|$, r ise uzaydaki bir kürenin yarıçapı olarak düşünülebilir. Eğer $r$ çok küçükse, tüm $X_{i}-X_{j}>r, \theta(z)=0, C(r, m)=0, C(r, m)=$ 0 , aksi takdirde $C(r, m)=1$. Çok büyük ve küçük $r$, durumunda sistem doğal özelliği yansıtamaz. Genellikle, $r$ değeri $0 \leq \mathrm{C}(\mathrm{r}, \mathrm{m}) \leq 1$ olmalıdır. $C(r) \sim \alpha r^{\gamma}$, $\alpha$ bir sabit, $\gamma$ ise korelasyon üsteli veya korelasyon boyutudur. ( $\log \mathrm{C}(\mathrm{r}), \log \mathrm{r}$ ) grafiğin eğimidir.

Eğer korelasyon üsteli doygunluğa ulaşıyorsa sistem kaotiklik sergiler. Eğer gömme boyutu s1nirsız artarsa sistem stokastiktir. 
Korelasyon boyutunun nasıl hesaplanacağını göstermek için, bir eğrinin uzunluğunu ölçmek istediğimizi varsayalım. Korelasyon boyutu $\mathrm{D}_{2}$ şu şekilde tanımlanır:

$$
D_{2}=\left|\frac{\lg \left(C_{2}(r, m)\right.}{\lg (r)}\right|=\mid \frac{\ln \left(C_{2}(r, m)\right.}{\ln (r)}
$$

Farklı m için hesaplanan korelasyon integralinin doğrusal parçalarının eğimleri regresyon analizi ile bulunarak $\mathrm{D}_{2}$ ler hesaplanır. m' ye bağlı $\mathrm{D}_{2}$ (m) doyum noktasına ulaştığında kaotik sistemin gömme boyutu belirlenmiş olur.

\section{B ITTCOIN FIYYAT VERILERINNIN ANALİİ VE MODEL OLUŞTUR- MA}

$\mathrm{Bu}$ bölümde Bitcoin verilerinin kaotik yapısı görsel olarak incelenmiş ve bulunan gömme boyutuna bağlı olarak, regresyon yöntemi ile fiyat tahmini için model oluşturulmuştur.

\subsection{Bitcoin Verilerinin Analizi}

Bitcoin fiyatlarındaki kaotik yapının incelenmesi amaciyla 2021 Şubat - 2021 Kasım döneminde günlük kapanış fiyatı ( \$ ) veri olarak kullanılmıştır. (URL-1,2021) Bu çalışmamızda kullanılan verilere ait grafik Şekil 1 de görülmektedir. Şekil 2 ve Şekil 3, zaman serilerinin karmaşık bir yapı sergilediğini göstermektedir. Ayrıca faz noktasının bir tür düzensiz hareketi vardır (Şekil 4). Verilen r için, denklem (2.3) temelinde bir $\mathrm{C}(\mathrm{r}, \mathrm{m})$ değeri elde ederiz. Doğrusal regresyon yöntemini kullanılarak denklem (2.4) temelinde her bir doğrunun eğimi hesaplanır. Korelasyon integralleri logaritmik eksenlerde belirli bir noktada doyuma ulaşmaktadır Şekil 2 de doğrunun eğiminin kademeli olarak arttı̆̆ını ve $\mathrm{m}$ değeri arttıkça eğrilerdeki mesafenin kademeli olarak azaldığını göstermektedir. Şekil 3 de m değeri $\operatorname{arttıkça~} \mathrm{D}_{2}(\mathrm{~m})$ değerinin kademeli olarak sabit hale geldiğini göstermektedir. Bu nedenle, çekici boyutu yaklaşı 1.62 dir ve doygunluk gömme

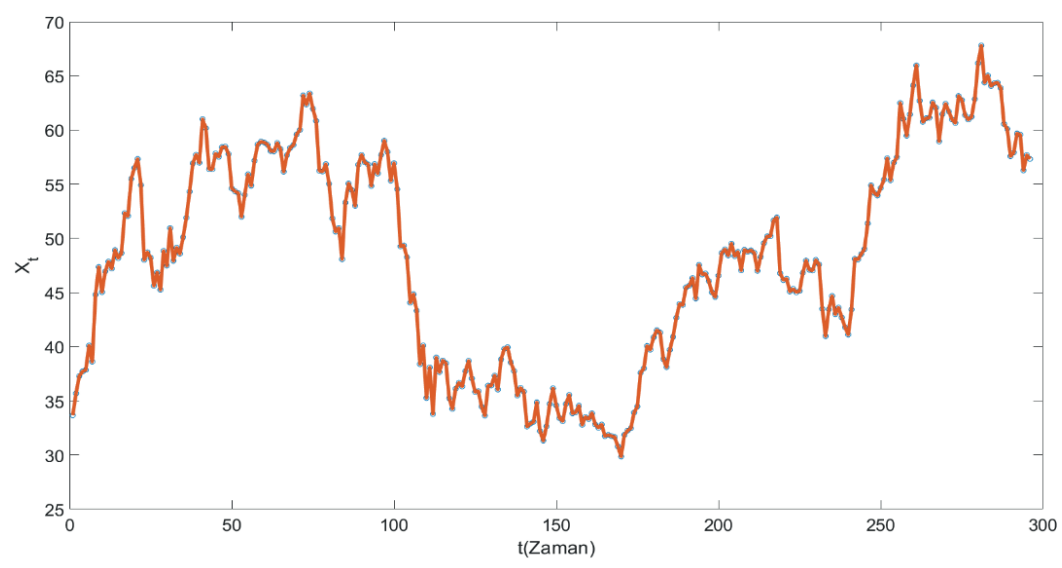

Şekil 1. Bitcoin günlük kapanış fiyatı verilerine ait seri

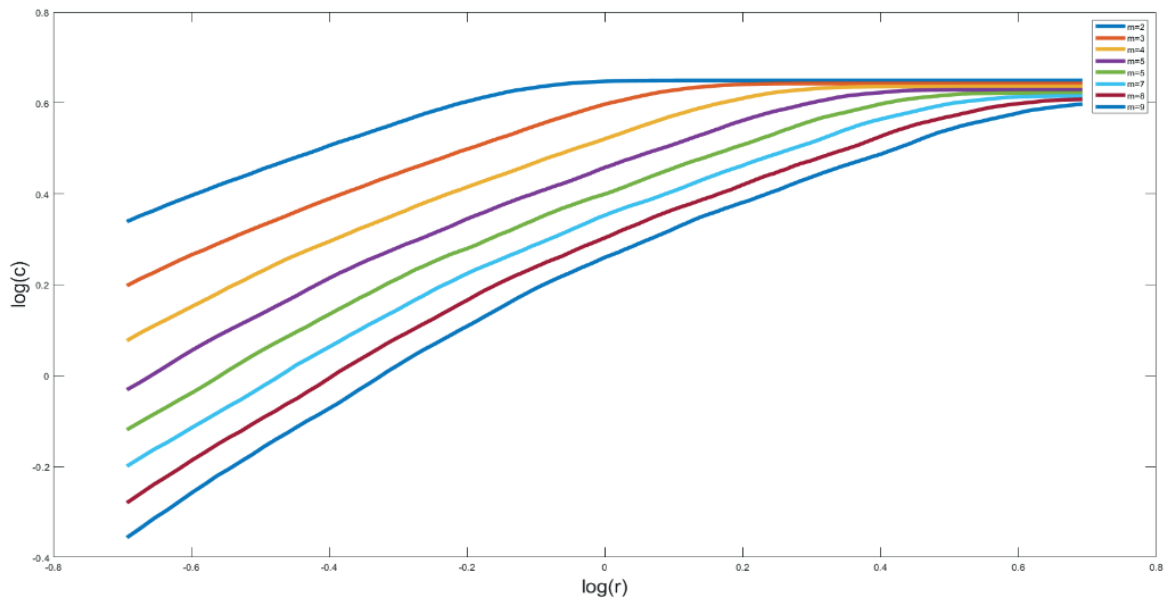

Şekil 2. Bitcoin serisi için korelasyon boyutu 
boyutu 4 olarak bulunmuştur. $\mathrm{D}_{2}(\mathrm{~m})$ değeri fraktal olduğundan, Bitcoin verilerinin garip çekici ve karmaşık yapıya sahip doğrusal olmayan kaotik bir sistem olduğu sonucuna varabiliriz.

\subsection{Model Önerisi}

Bitcoin verilerinin kaotik davranışı incelenmesi sonucunda gömme boyutu 4 olarak bulunmuştur. Bu bize Bitcoin günlük kapanış fiyatlarının tahmin edilebilir boyutlarda olduğunu göstermektedir. Gömme boyutuna bağlı olarak regres- yon analizi yöntemi ile aşağıdaki tahmin modelini kuralım;

$$
x_{t}=c_{0}+c_{1} x_{t-1}+c_{2} x_{t-2}+c_{3} x_{t-3}+c_{4} x_{t-4}+\varepsilon
$$

X bağımsız değişken matrisi, Y bağımlı değişken matrisi olmak üzere

$\mathrm{C}=\left(\mathrm{X}^{/} \mathrm{X}\right)^{-1} \mathrm{X}^{/} \mathrm{Y}$ yansiz tahmincisiyle modele ait katsayılar $C_{0^{\prime}}, C_{1}, C_{2}, C_{3}, C_{4}$ hesaplandı̆̆ında tahmin modeli aşağıdaki şekilde oluşturulur.

$x_{t}=0.6854-0.0039 x_{t-1}-0.0132 x_{t-2}+0.0648 x_{t-3}+0.9366 x_{t-4}+\varepsilon$

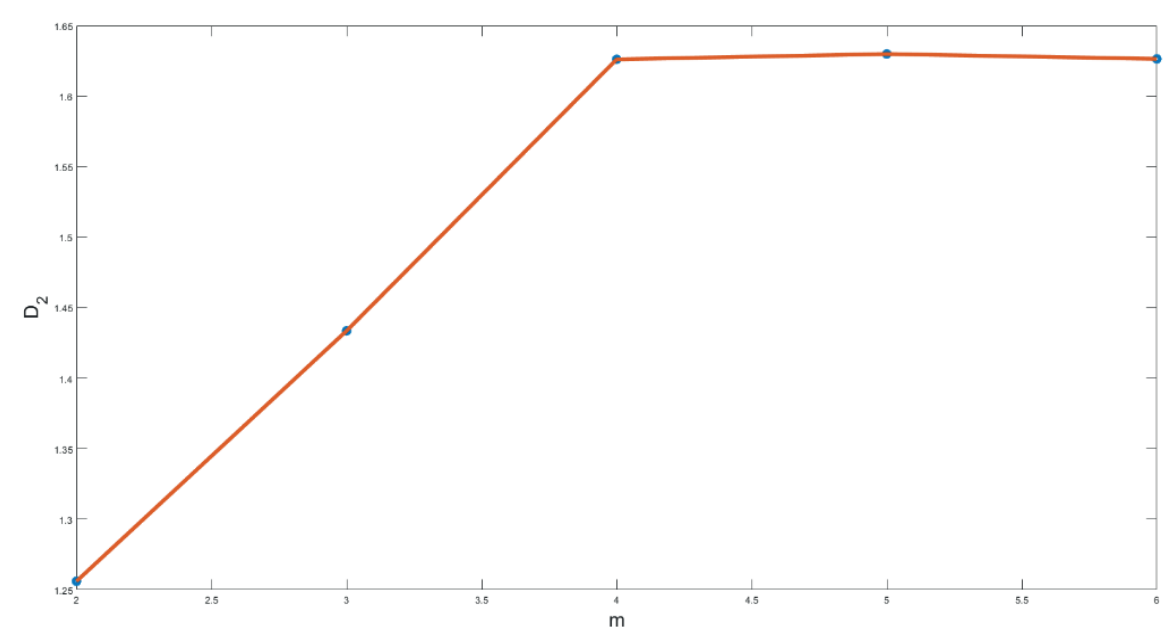

Şekil 3. Bitcoin serisi için gömme boyutu

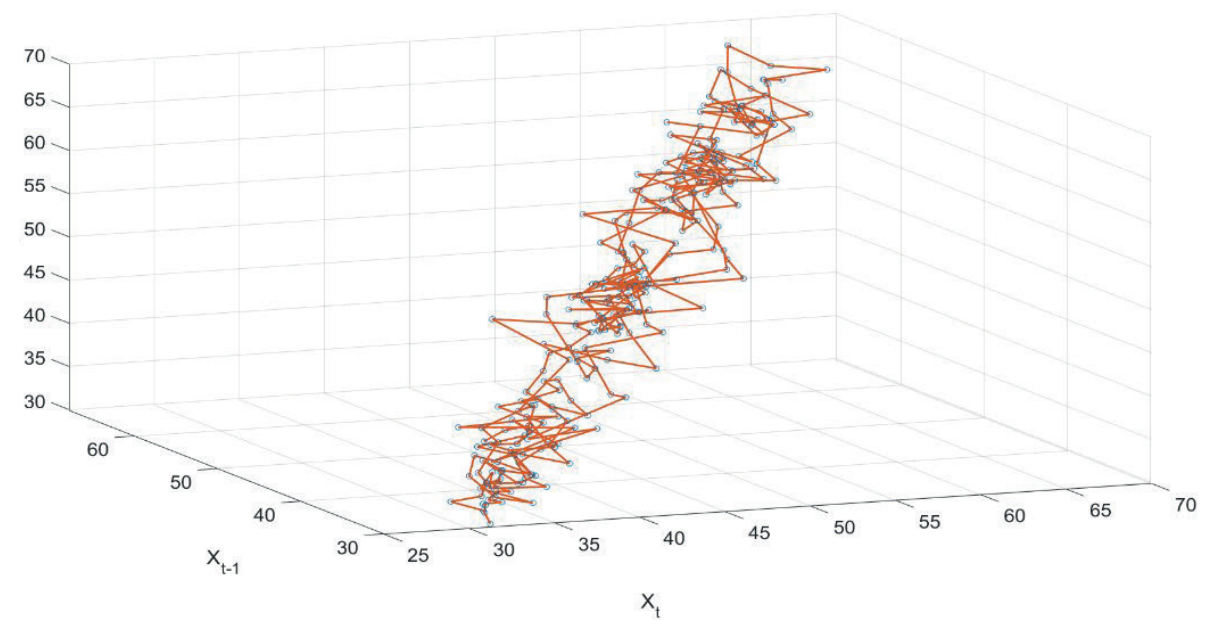

Şekil 4. Bitcoin serisi için 3-D Faz uzayı 
Bu modelin hata analizine ait grafik Şekil 5 de sunulmuştur. Ayrıca Bitcoin tahmin değerlerine ait grafik Şekil 6'da verilmektedir. Hata(Error) grafiğinin rassal davranışlı olması modelin uygunluğunu göstermektedir.

\section{SONUÇ}

Bu çalışmada Bitcoin verilerinin (URL-1,2021) davranışları incelenmiştir. Korelasyon integrali yöntemi kullanılarak gömme boyutu belirlenmiş ve Bitcoin tahminlerinin öngörülebilir boyutta olduğu gösterilmiştir. Gecikmelere bağlı olarak kurulan model regresyon yöntemiyle belirlen- miştir. Bu çalışmaya bağlı olarak farklı otoregresif modeller, stokastik modeller kullanılarak yeni çalışmalar yapılabilir. Bu çalışmadaki tüm analizler MATLAB kod yazılarak gerçekleştirilmiştir.

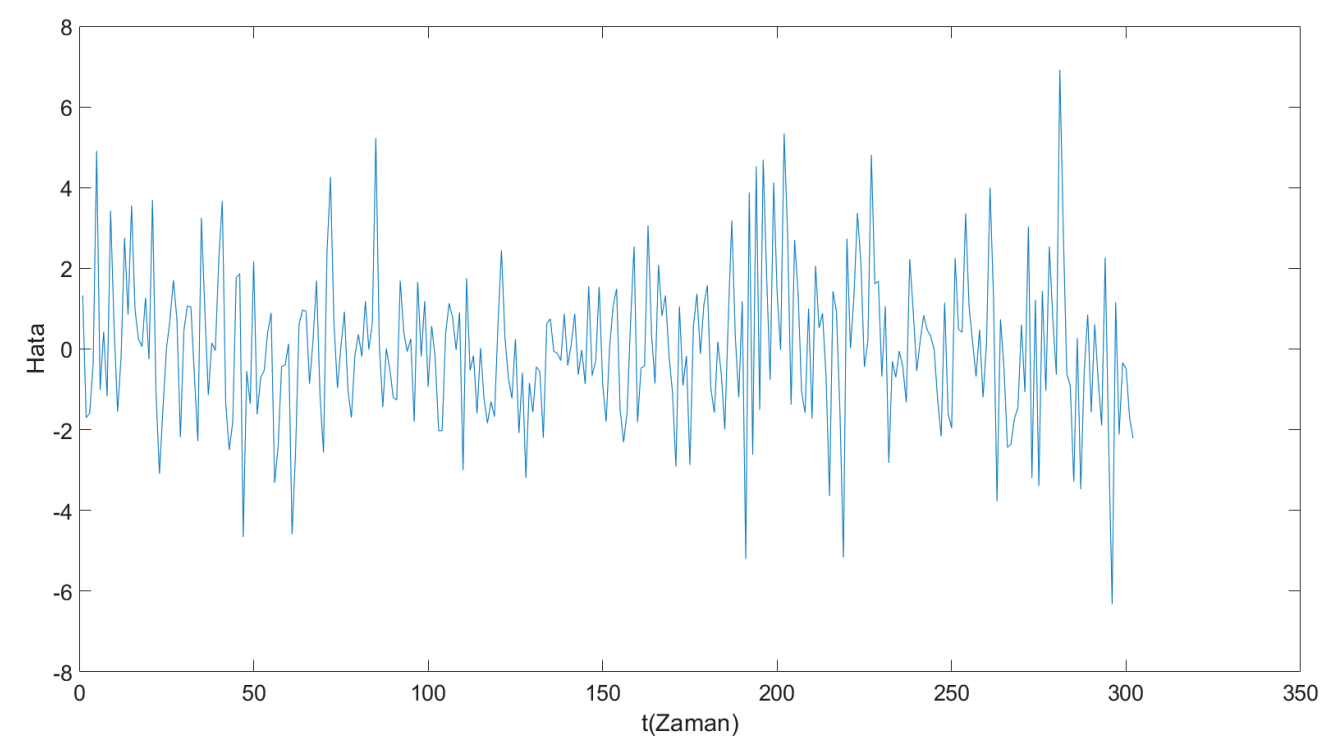

Şekil 5. Hata analiz grafiği

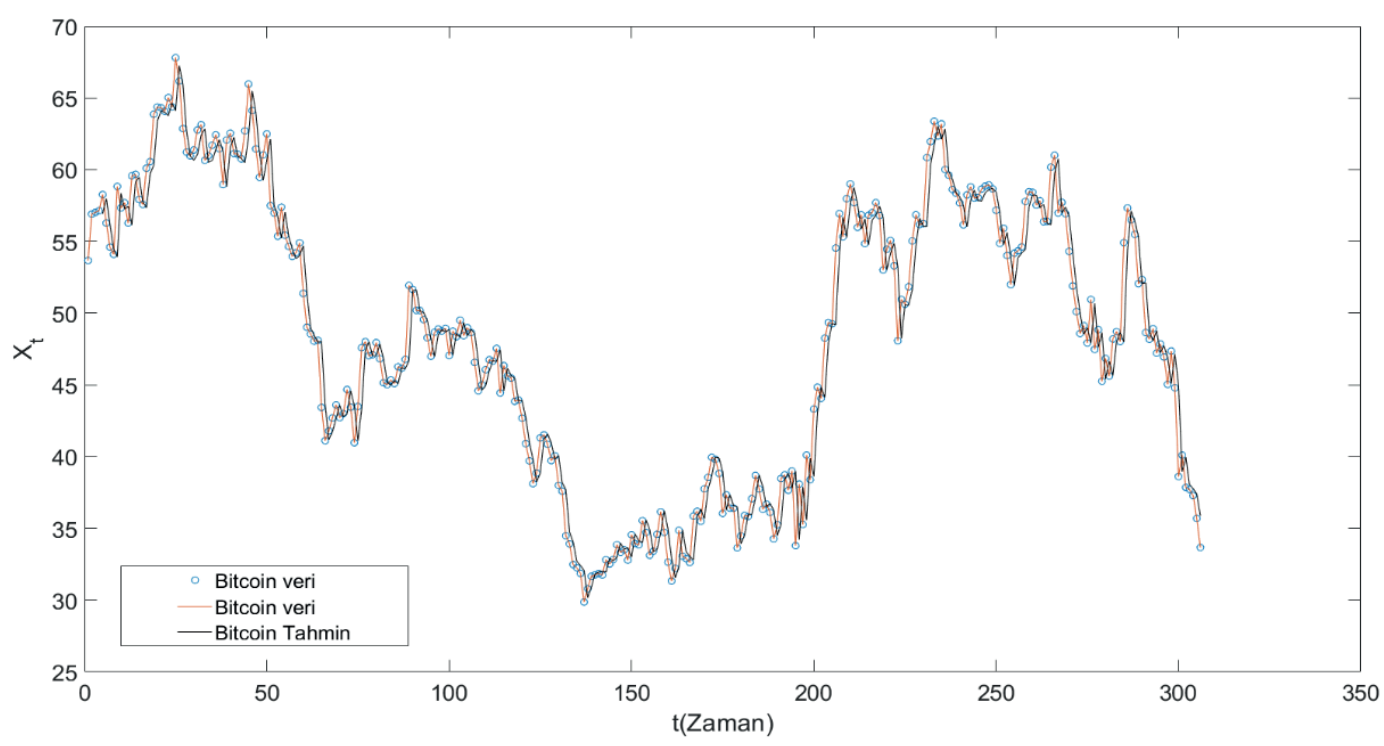

Şekil 6. Bitcoin tahmin değerleri grafiği 


\section{KAYNAKÇA}

ALBOSTAN, A. ÖNSÖZ, B. (2015). Günlük Akarsu Akımlarının Kaotik Analizinde Dalgacık Yaklaşımınin Uygulamasi Journal of the Faculty of Engineering and Architecture of Gazi University. 30( 1), 39-48.

BOEING,G. (2016). Visual Analysis of Nonlinear Dynamical Systems:Chaos, Fractals, Self-Similarity and the Limits of Prediction. Systems,4(4),37.

CLARK, A. (2006) .Modelling the net flows of U.S. mutual funds with stochastic catastrophe theory .The European Physical Journal B.50, 659-669.

GANGOPADHYAY, P., ELKANJ, N., RAHMAN, M. A. (2011). Application of Theories of Complexity and Chaos to Economic Misgovernance. Journal of Mathematics and Statistics.7(3):239-248.

GRASSBERGER P AND PROCACCİA I. (1983). Dimension and entropies of strange attractors from a fluctuating dynamics approach Physica.D,13,34-54.

ISERİ, M., CAGLAR, H.,CAGLAR,N. ( 2008.) A model proposal for the chaotic structure of Istanbul stock exchange, Chaos, Solitons \& Fractals, Elsevier. 36(5):1392-1398

NICHOLS, NANCY A. (1993). Efficient? Chaotic? What's the New Finance?. Harvard Business Review.71(5):0017-8012.

SAKIZ,B. \& GENCER,A.H. (2017).Yapay Sinir Ağları ile Bitcoin Fiyatını Tahminleme. International Confrerence on Eurasian Economics.

SLOAN, K.,(2011). Viewing Organizations Through the Lens of Chaos Theory: Thoughts on Applicability and Usefulness. European Journal of Social Sciences.21(3).

TAKENS F. (1981).Detecting strange attractors in turbulance. New-York: Springer-Verlag. pp .366-381.

PETERS,E. (1991). Choas and order in the capital markets. New York: John Wiley\&Sons Inc.

URL-1, https://www.marketwatch.com

URL-2, https://www.bitcoin.org 